\title{
Molecular Dynamics of the Transport of Ions in a Synthetic Channel
}

\author{
D.A. Morton-Blake \\ School of Chemistry, Trinity College, Dublin 2, Ireland \\ tblake@tcd.ie
}

\begin{abstract}
Keywords: Ion channels, Molecular dynamics, Ion migration, Synthetic membrane, Electric fields, Rigid and flexible channels, Oscillating trajectories, Molecular sliding friction.
\end{abstract}

\begin{abstract}
Molecular dynamics investigations of ions in certain non-bulk media predict that they are capable of significantly greater mobilities than when in the liquid state. The entries of $\mathrm{Li}^{+}, \mathrm{Na}^{+}$, and $\mathrm{K}^{+}$ions from electrolyte media into proposed synthetic channels consisting of fourteen 15-crownether-5 (CE) rings bonded in stacked conformations are described and their subsequent dynamics in the channel discussed. The importance of channel flexibility is established by investigating two CE channels that are structurally similar but vary in the rigidity with which their rings are connected. The dynamics of cation channel migrants are simulated across a bilayer membrane between two bulk aqueous salt solutions and also when the channel floats freely in an aqueous medium. Various features of ion behaviour are investigated in the presence, and in the absence, of an electric field applied along the channel axis. The novel oscillatory behaviour of the ions in the channel is investigated, together with the possibility of their exits into the liquid medium. The frictional forces opposing the ion trajectories are calculated, found to be $\sim 10 \mathrm{nN}$ and attempts to formulate frictional laws for nanoscale systems are discussed.
\end{abstract}

\section{Introduction}

The passage of ions across membranes is central to the functioning of living cells and has been extensively studied [1]. However the mechanisms describing these processes are not necessarily confined to biological processes; current development of molecular mechanical devices operating on a nanoscale [2] show that the elucidation of the mechanisms of cellular ion transport would be essential in the design of nanodevices performing analogous ion transport in an entirely synthetic environment.

Although there have been many interesting molecular dynamics simulations of ion migration in biological transporters [3], an understanding should be sought of the fundamental principles and modes of action underlying the mechanisms occurring in the motion of small molecular units through matter. The systems to be described will correspond to single-channel currents in which the complexities of the molecular channels have been sufficiently reduced to facilitate the study of the dynamics of the overall process on as fundamental a level as possible. Another reason for selecting a structurally simple channel as a theoretical model is that it allows the effects of altering a desired feature that governs the passage of the ion in relative isolation. It is hoped that the understanding of the transport processes thereby gained might ultimately be useful in the design and synthesis of nanochemical devices that can move small molecules and ions between sites within an artificial structure. Some of the approaches taken include the emulation of natural processes by applying molecular dynamics to a system in which ions move through a synthetic molecular channel that is embedded in a simple amphiphilic bilayer partitioning the two aqueous media electrolytes. Another study makes an effort to elucidate features describing the ions as they attempt to enter the mouth of the channel, followed by that of their subsequent dynamic behaviour within the channel.

Several types of ion channel transporters have been synthesized to perform experiments to investigate the trans-membrane passage of ions [4]. Requirements of the channel's molecular

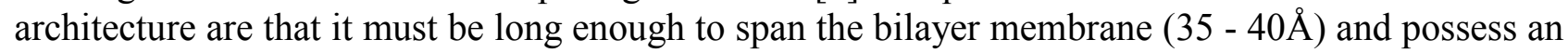
adequate internal diameter for the passage of the ions. It should also be amphiphilic so that its ends 
(each of which contacts an aqueous ionic medium) is hydrophilic, its exterior (embedded in the hydrocarbon tails of the bilayer) is hydrophobic, while the interior, through which the ions migrate, is hydrophilic. Notable examples are cyclodextrins [5], self-assembling peptide nanotubes [6], helix-forming oligopeptides such as gramicidin [7], macrocyclic compounds that are represented by crown ethers, aza-crown ethers and hydraphiles [8].

The transport of ions across cell membranes may occasionally be passive, in which case they are independent of the dynamics of the transporter, but require there to be a concentration gradient of the migrating ions in the direction of the migration; this implies diffusion via an electrochemical gradient. More commonly however, transport in natural processes is active, the transporter becoming an ion pump that is driven by an external energy source such as the reduction in free energy that occurs in ATP hydrolysis. This feature may be communicated as dynamical features to the channel or to a channel-opening/closing ion sensor; it could also transport ions along a positive concentration gradient.

The channels that are selected for our study are necessarily passive channels, but some features of their dynamics will emerge. In our investigation we shall use molecular dynamics to study the motions of the ions as they approach and enter the channel from the aqueous medium, their dynamics within the channel (together with the motions of the atoms of the channel) and their exit as they re-emerge into the aqueous solution. Since our intention is to explore novel features for possible application in nanoscience, in the present work we propose channels which, without the presence of ion/solvent selectivity components found in natural ion channels, must perform any necessary selection using their molecular structures.

The nanosecond time intervals over which molecular dynamics can currently be conducted is much shorter than the timescales of natural ionic processes. As a result, it is sometimes necessary to boost the particle dynamics by appropriate means. Consequently in some of the investigations described a static electric field has been applied along the axis of the ion channel. This practice could justifiably be described as a natural one in view of the sometimes large fluctuating electric fields generated at local points of interest by the motions of ionic and molecular species.

\section{Molecular structures and methodologies}

\section{Channel}

The ion channel to be described in this investigation is a proposed oligo-crown ether molecule consisting of fourteen 15-crown-ether-5 (CE) monomers shown in Fig. 1 which are covalently bonded into a stacked configuration at carbon atoms labelled ' $*$ ' in (a) and (b). This makes a channel length of $35.0 \AA$, which is close to the thickness of a phospholipid bilayer membrane. In addition, each of the two terminal rings on both species of channel has five $\mathrm{OH}$ substituent groups. One purpose of these groups is to explore a further degree of dynamic flexibility on the end rings; the present work will show that the end rings play a role in the admission of $\mathrm{Na}^{+}$ ions into the channel. A secondary purpose is to make these rings hydrophilically compatible with aqueous solutions on either side of the bilayer membrane.

Parts (b) and (c) of Fig. 1 show that the 14 CE rings, whose smallest diameter is just over 4 $\AA$ diameter, partition the channel into a series of cavities with minimum diameter of about $7 \AA$. Previous molecular dynamics studies [9] showed that the channel permitted the passage of cations but that the rings were too narrow to admit solvent water molecules from the external aqueous ionic solution. Anions, too, were denied entry even in the presence of strong electric fields along the channel axis. In investigations of the entry and passage of the ions the CE channel was allowed to float freely in the aqueous medium. In order to examine the dynamics in the oriented channel (sometimes in the presence of an electric field), and to permit statistical interpretations from a system containing several channels, a membrane-embedded model was defined to allow molecular dynamics to be performed on the 25-channel $5 \times 5$ lattice shown in Fig. 1(d). 


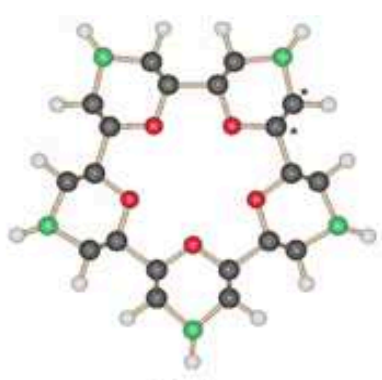

(a)

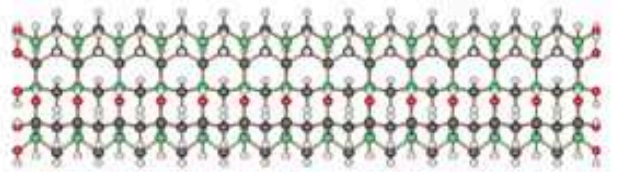

(c)

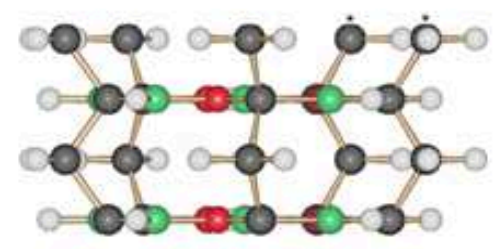

(b)

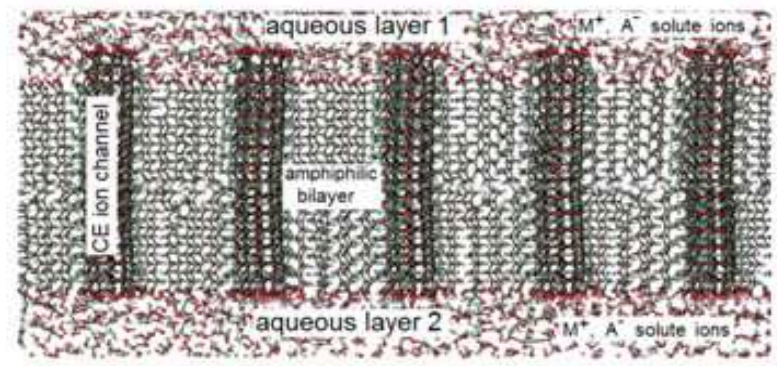

(d)

Fig. 1 (a) to (c) A rigid oligo-crown-ether ion channel in which each CE monomer is linked to an adjacent one at the 10 carbon atoms labelled '*'. Black atoms are carbon, red are oxygen and green are nitrogen. Part (d) represents a 2-dimensional $5 \times 5$ 'lattice' of 25 channels spanning a bilayer membrane partitioning two aqueous salt layers.

\section{Molecular dynamics}

Molecular dynamics calculations were performed using DL_POLY [10] together with custom-written programs for data preparation and processing. The partial atomic charges in the channels (referred to in the previous section) were obtained from Restricted Hartree-Fock (RHF) calculations deploying the Gaussian package [11] using 6-311 basis sets supplemented by $(2 p, 2 d$, $3 d$ and $3 f$ ) polarization functions. These were performed on model molecules consisting of $-\mathrm{CH}_{2}-$ or (-CH-NH-) -linked 5-CE-15 rings, hydrogenated where necessary. The resulting charges were compared with those obtained from calculations on smaller molecules (acetic acid and dimethylamine $\mathrm{H}_{3} \mathrm{C}-\mathrm{NH}-\mathrm{CH}_{3}$ ) on which Møller-Plesset (MP2) corrections had been performed. The consistency between the latter and the RHF results was deemed to be sufficient to accept the RHF charges without adjusting their values other than the minor ones resulting from dehydrogenating the 2-ring model molecules that had been deployed as models for the quantum calculations. Molecular geometries were calculated by the same methods on model compounds.

The molecular dynamics computations were conducted under both constant volumetemperature and constant pressure-temperature conditions (Evans NVT and Hoover NPT thermostats). Simulations of a million time-steps, where a time-step was 1 femtosecond $\left(10^{-15} \mathrm{~s}\right)$, were first performed to ensure that the molecular dynamics system had achieved thermal and dynamic equilibrium, while the running times for the migrations of the ions in the channel could be either longer (as when runs of 10 million time-steps were used to investigate the possibility of more ions entering the channel) or much shorter (a few thousand time-steps, when investigating the behaviour of single ion species in the channel). The simulation box consisted of a $36 \times 36 \times 75 \AA$ cell containing the 685 -atom channel (Fig. 1) in an aqueous solution of various molarities ( 0 to $5 \mathrm{M})$ in $\mathrm{NaCl}$, about 3500 water molecules and a varying number of ions depending on the salt concentration. The timestep interval for most of the simulations was $1 \mathrm{fs}\left(10^{-15} \mathrm{~s}\right)$ but in one exercise to be described, that involved fast processes, this was reduced to $0.1 \mathrm{fs}$ in order to ensure equilibrium in the short time intervals that were involved in the exercise. 
For the 2- and 3- body interactions involving the bonding- and non- bonding channel atoms, the atomistic potentials of Åquist [12] were used for $\mathrm{Na}^{+}$and those of Lee and Rasaiah [13] for $\mathrm{Cl}^{-}$; DREIDING (6-12) potentials [14] were used for the remaining atom pairs. As the use of these potentials, together with those of Berendsen's SPC model [15] successfully simulate the solvation of the ions by the water molecules no additional electronic polarization effects were allowed for (which in any case would be inconsistent with the use of the atomistic potential described). Potential functions for heteroatomic pairs were calculated by the geometric-mean rule.

\section{Results of simulations}

\section{Atomic coulombic contributions}

The dynamics of the migrating ions are sensitive to the coulomb forces within the channel. These involve the electric field which is generated by two sources: (A) an unequal density of charges $\rho_{u}$ and $\rho_{l}$ in the 'upper' and 'lower' aqueous layers (as produced by the ions in these regions) and (B) the partial atomic charges of the atoms constituting (particularly) the hydrophilic interior of the channel. From the charge neutrality of the complete system it can be shown [16] that the axial field $\mathcal{E}_{z}$ is independent of the detailed distribution of the charges in the channel's terminal regions and is given by $\varepsilon_{z}=\sigma / \varepsilon_{0}$, which is the same as that produced by a plane surface bearing a surface charge $\sigma$.

The electrostatic potential in (B) is calculated from the atomic geometry of the 14 CE rings, their spacings and a knowledge of the atomic charges $q_{j}$ in the channel, and the relation:

$$
V(z)=\sum_{i=1}^{14} \sum_{j=1}^{n} \frac{q_{j}}{4 \pi \varepsilon_{0} \sqrt{r_{j}^{2}+\left(p_{i}+z\right)^{2}}}
$$

where $n$ is the number of atoms in each of the 14 component CE rings, $r_{j}$ is the distance of charge $q_{j}$ from the axis in the ring and $p_{\mathrm{i}}$ is the axial coordinate of the centre of the $i^{\text {th }}$ ring. The atomic charges $\left[q_{j}\right]$ are calculated by quantum chemical methods described in Section 2.2 and the geometric parameter sets $\left[r_{j}\right]$ and $\left[p_{j}\right]$ are the result of averages taken over the atomic positions during a molecular dynamics simulation. Values of the resulting channel axial electrostatic potential $V(z)$ in Fig. 2 show that they decrease to below $-10 \mathrm{v}$ in most of the channel region. Also shown in the figure are the potentials obtained when the channel, separated from the membrane, is immersed in a $3 \mathrm{M}$ aqueous $\mathrm{NaCl}$ aqueous solution and molecular dynamics are performed for $10^{6}$ time-steps. This calculation, which includes the atomic charges in the surrounding atomic species from the solvent and ions, results in potentials which are a little more negative than those obtained from the channel alone. This effect which, together with the peaks outside the channel, does not disappear by dynamical averaging even after 5 million time steps, seems to be a consequence of the longer relaxation times (or partial immobilization) of solvent molecules and ions near the walls of the channel walls and particularly near its ends, which Fig. 2 shows to be a region of negative potential. After emerging from the channel's central -10 to $-12 \mathrm{v}$ coulomb potential region into the external aqueous medium a cation would encounter local coulomb energy wells as it becomes hydrated by solvent molecules (which we shall see cannot enter the channel). Consequently the walls of the potential well constitute an energy barrier to a $\mathrm{M}^{+}$ion migrating either into or out of the channel.

It may be interesting to note that a mathematical model in which the charges of the atoms within the channel are represented by uniformly charged cylinders with radius $r$ from $z=0$ to $z=L$ with surface densities $\sigma$ produces axial potentials [16] with values closely similar to those obtained using Eqn. (1) 


$$
V(z)=\frac{\sigma r}{2 \varepsilon_{0}} \ln \left|\frac{1+Y_{l}}{1-Y_{l}}\right|_{l=0}^{l=L}
$$

from each cylinder

where

$$
Y_{l}=\tan \left(\frac{1}{2} \tan ^{-1} \frac{z+l}{r}\right)
$$

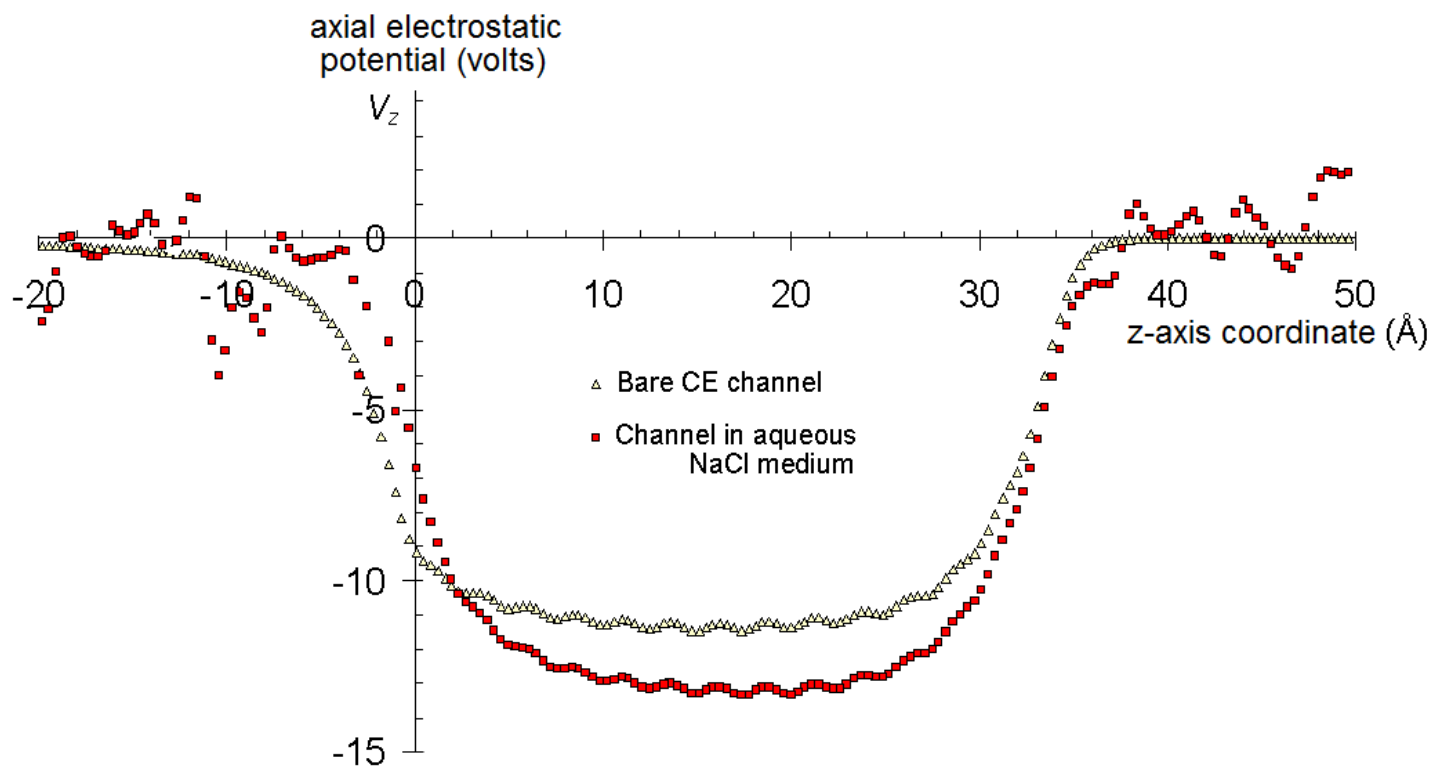

Fig. 2 The electrostatic potential along the axis of a CE channel with and without the inclusion of the atomic charges in an external concentrated $\mathrm{NaCl}$ solution. The extent of the channel along the $z$ axis is 0 to $35 \AA$.

but without the 'ripples' in the Fig. 2 traces caused by the individual CE rings in the atomistic calculation.

Despite the negative potential along the channel's axis, the entry of a cation into the channel is not necessarily a spontaneous process as, in order to surmount the barrier, the ion may have to await the advent of favourable factors concerned with the ions' positions and momenta near the mouth of the channel over the time interval considered.

\section{Lattice of channels}

In the 25-channel unit-cell microlattice shown in Fig. 1(d) in which the electrolyte solute was $\mathrm{LiF}, \mathrm{NaF}$ or KF, we placed individual ion species 'flush' with one end of the channel and used molecular dynamics to monitor the motions of the ions and solvent molecules. The particle trajectories are shown in Fig. 3. Only the cations $\left(\mathrm{Li}^{+}, \mathrm{Na}^{+}, \mathrm{K}^{+}\right)$can enter the channel. The internal diameter of $\sim 4 \AA$ is too small to admit water molecules, and $\mathrm{F}^{-}$(whose anion radius is actually smaller than that of $\mathrm{K}^{+}$) is also denied entry, showing that it is the coulomb forces that are dominant in deciding the admission of the ions. We conclude that the strongly negative coulombic axial potential (CE rings' $\mathrm{O}$ atoms) makes the channel a cation trap. After their initial oscillations the $\mathrm{M}^{+}$ migrants come to rest in specific 'halting sites' in the channels. Atomic 'snapshot' plots, inspection of their coordinates and (Section 3.4 below) radial pair distribution functions show that the sites are within $1 \AA$ of the axis and up to about $1 \AA$ from the planes of the ether $\mathrm{O}$ atom. In order to determine the possibility of ion mobility between the sites, a moderate electric field of $0.1 \mathrm{v} \AA^{-1}$ was applied to an aqueous LiF solution. Fig. 3(d) shows that the field displaces some of the $\mathrm{Li}^{+}$ions to downfield 
sites. Note the oscillations in the ion trajectories when the ions initially enter the channel. This feature will be discussed below.


(d) $\mathrm{Li}^{+}$

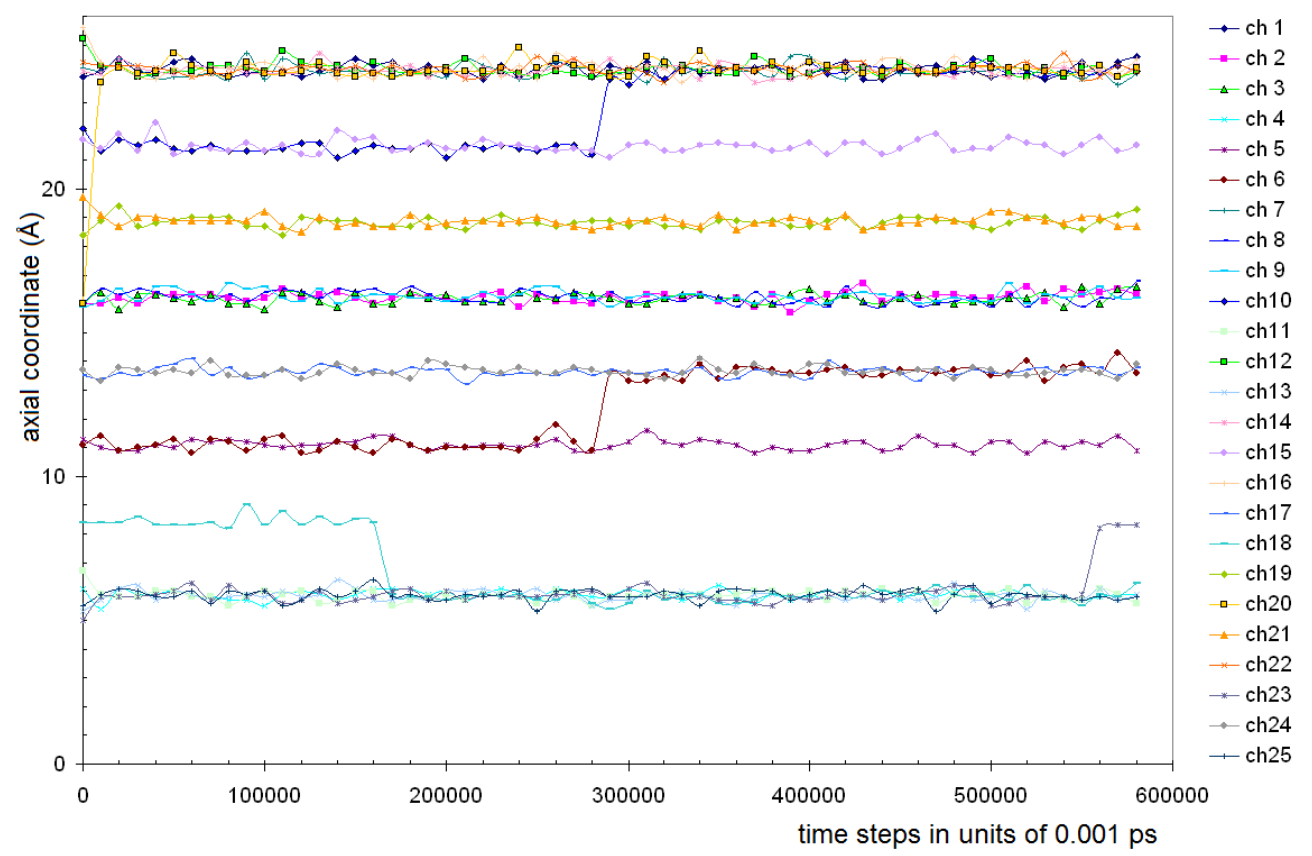

Fig. 3(a) to (c) The trajectories of cations that have entered the channel from aqueous solutions of $\mathrm{LiF}, \mathrm{NaF}$ and KF. (d) The trajectories of the $\mathrm{Li}^{+}$ion in the 25 channels (ch1 to ch25) after longer times in the presence of an axial electric field. The traces are for the 25 channels in the bilayer unit cell sketched in Fig. 1(d).

The behaviour of multiple ions in a single, solvent-immersed channel

In order to examine details of the entry of the ions in the channel, we now confine our attention to a single channel which, freed from the membrane, is henceforth a solute in the aqueous bulk. The 'desolvation' energy barrier at the channel end (Section 3.1) delays the entry of the cation and usually its trajectory could not be investigated in a period that is appropriate to the molecular dynamics timescale. In order to obtain results in a tractable time we had to ensure that there were a number of ions sufficiently near a channel end so that they could be deemed to have already surmounted the barrier. 


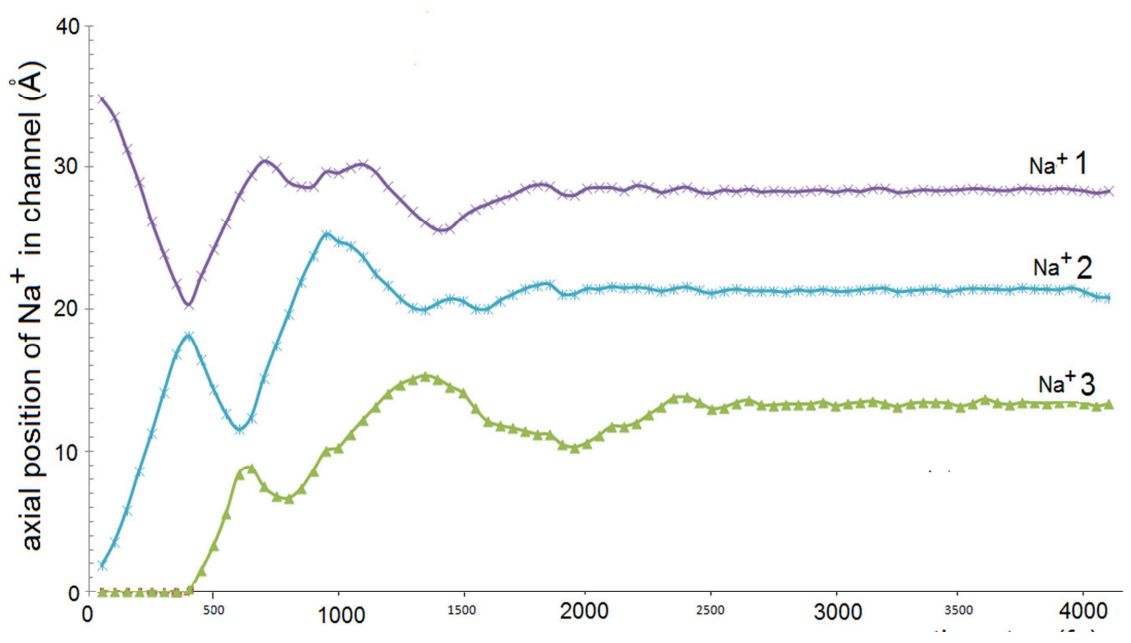

Fig. 4 The axial trajectories of three $\mathrm{Na}^{+}$ions from a $3 \mathrm{M}$ solution and their interactions in the channel. Their mutual interactions are observable and are discussed in the text.

While the Figure shows that a small number of $\mathrm{Na}^{+}$ions enter the channel almost immediately, after $10^{5}$ time-steps the remainder still remained outside. Because of coulombic restrictions it would appear that no more than three cations could enter the channel at any one time. Of these three entrants, which take up final positions that are roughly evenly distributed along the axis, Fig. 4 shows that ion $\mathrm{Na}^{+} 1$ enters from the 'top' of the channel $(z=35 \AA)$ and $\mathrm{Na}^{+} 2$ from the 'bottom' ( $z=0 \AA$ ). The Figure shows the migrating ions' interactions: in their mutual encounters, these two ions initially repel each other at time 400 fs before reversing their directions; ion $\mathrm{Na}^{+} 2$ then repels ion $\mathrm{Na}^{+} 3$ which has just entered the channel. We find that as they experience a large negative electrical potential along the axis, as well as an energy barrier at the channel end, the cations do not spontaneously exit the channel.

When the first migrant enters the channel the linear momentum conferred by its 'drop' into the axial low-energy region allows the ion to partially scale the opposite wall of the energy trough. It then comes to rest in one of the CE ring 'halting sites' that were described in a forgoing subsection.

\section{Single ions in a channel}

The upper curve in Fig. 5, which records the ion's axial position, shows that on reaching the $14^{\text {th }} \mathrm{CE}$ ring the ion rebounds and briefly oscillates between rings 11 and 13 and remains in the channel cavity (Section 2.1) bounded by rings 12 and 13, for the remainder of the investigation interval. The lower curve, which has the same time axis as the upper one, monitors the simultaneous radial displacement from the channel axis. An ion showing unhindered migration along the channel would not depart appreciably from the axis; the latter displacement would be small. The trajectory monitored by Fig. 5 shows a lateral displacement of $4 \AA$ before the ion reverses its progress along the channel and enters one of the molecular cavities between the $\mathrm{CE}$ rings. 


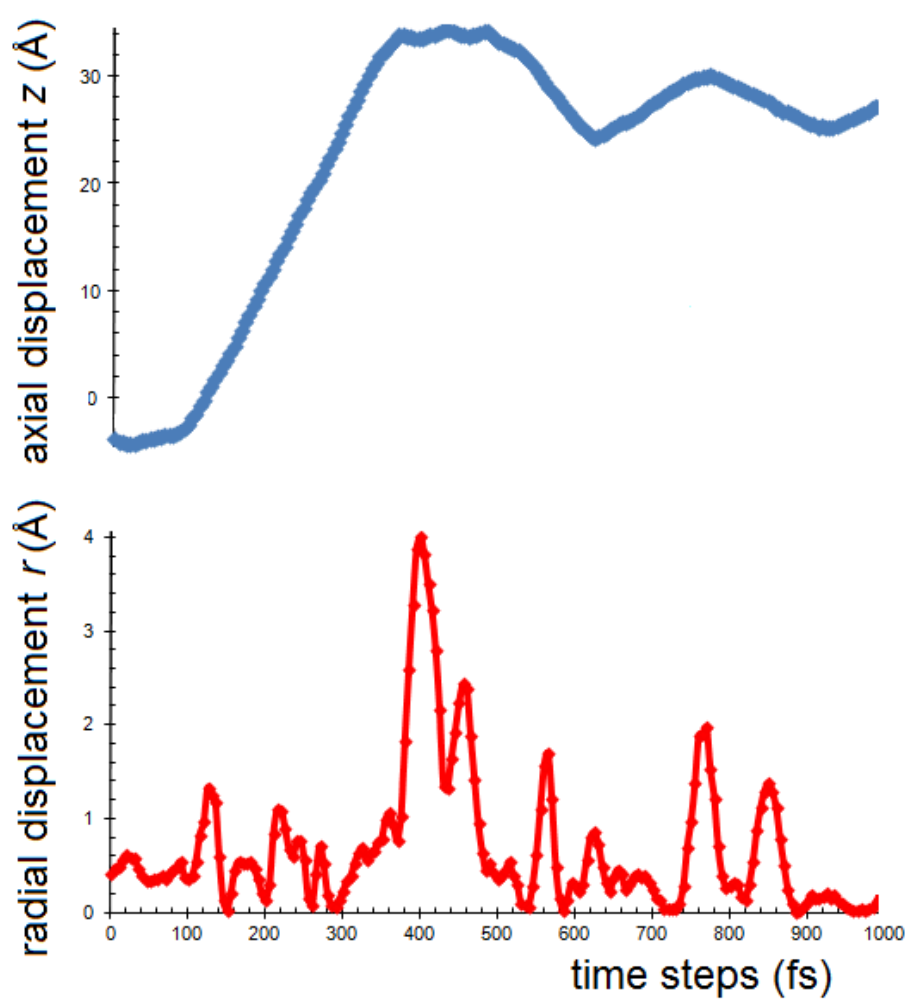

Fig. 5 The trajectory of a $\mathrm{Na}^{+}$ion in the channel in an axial electric field $(\varepsilon=0.5)$. The two simultaneous displacements are displayed along the $z$ axis (upper trace) and radially (lower trace).

At the conclusion of the dynamics of the migrant ions, the location of their subsequent 'halting sites' in the channel is provided by the radial pair distribution function for the migrant $\mathrm{Na}^{+}$ ion and the atoms of the $\mathrm{CE}$ ring shown in Fig. 6. The ether oxygen of the channel was distinguished from the $\mathrm{O}$ atoms of the water by declaring it as a separate particle species in the molecular dynamics; the latter interactions for the water interactions therefore do not appear in the figure.

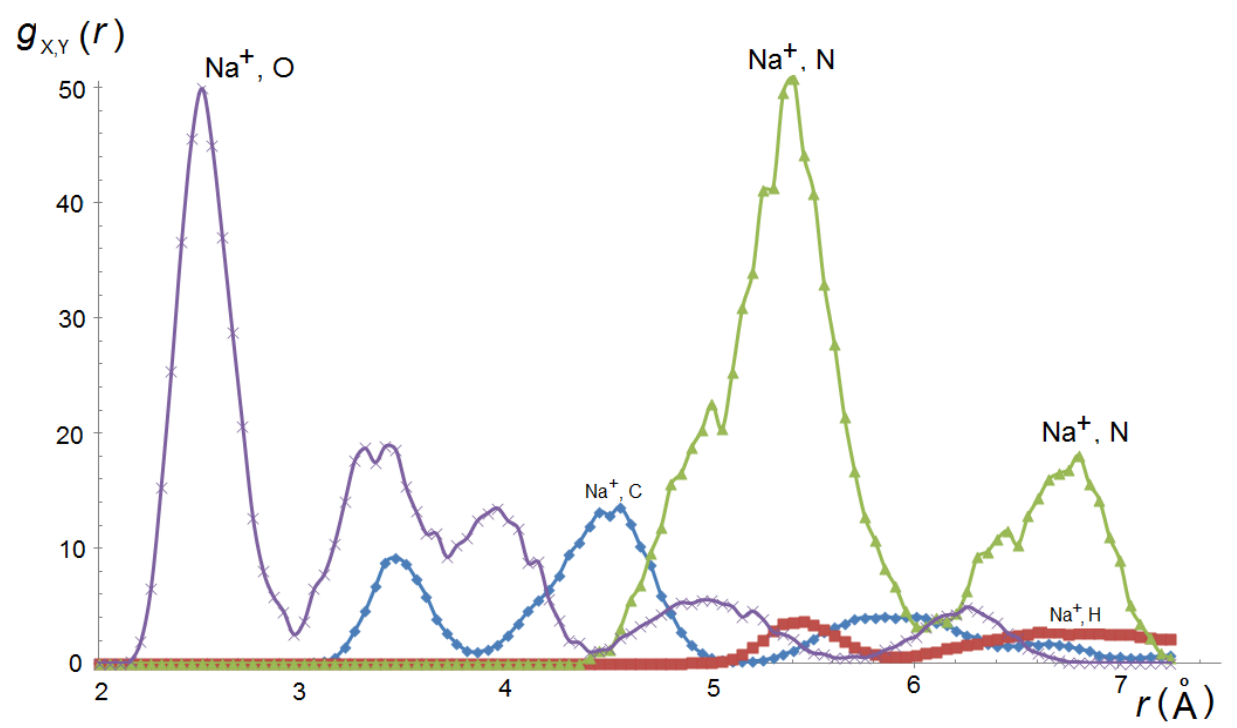

Fig. 6 Radial pair distribution functions between the $\mathrm{Na}^{+}$ion migrant and channel atoms.

The prominent peak at $r=2.5 \AA$ indicates the distance to which $\mathrm{Na}^{+}$ions within the channel approach the $\mathrm{CE}$ oxygen atom. Steric considerations preclude accessibility of these oxygen atoms 
to ions which are outside the channel to this distance. The $r$ value is a little shorter than the $2.63 \AA$ between $\mathrm{Na}^{+}$ion and a hydrating oxygen atom in the bulk $\mathrm{NaCl}$ solution. From the rdf peaks and the molecular geometry of the channel we see that the $\mathrm{Na}^{+}$ions settle into a halting site $0.9 \AA$ from the centre of a CE ring. The substantially larger values of the $r$ values of the $\mathrm{g}_{\mathrm{Na}}{ }^{+} \mathrm{O}(r)$ peaks than the $\mathrm{g}_{\mathrm{Na}}{ }^{+}, \mathrm{N}(r)$ is consistent with the greater proximity of the $\mathrm{Na}^{+}$site to the ether $\mathrm{O}$ than to the peripheral $\mathrm{N}$ atom.

\section{Electric fields and temperature}

Fig. 7 shows a $\mathrm{Na}^{+}$trajectory in a field of magnitude $\varepsilon=0.3 \mathrm{v} \AA^{-1}$. After 'dropping' through the $36 \AA$ length of the channel its direction is reversed by the energy barrier at the opposite end. The mutually opposing effects of the channel's internal $-12 \mathrm{v}$ potential and the applied field produce a series of oscillations that are rapidly damped from their initial $15 \AA$ amplitudes as the $\mathrm{Na}^{+}$ion is trapped at one of the cavity sites.

The cusp-like maxima of the oscillations in the Figure are due to the steep wall of the coulombic potential near the end of the channel that is furthest from the ion's entry position. Electric fields of lower intensities would bring the $\mathrm{Na}^{+}$ion to rest at other sites of the channel (the flat portion of the coulomb basin). The atomic sliding friction that causes the dampening of the oscillations is discussed in Sections 3.9 and 3.10.

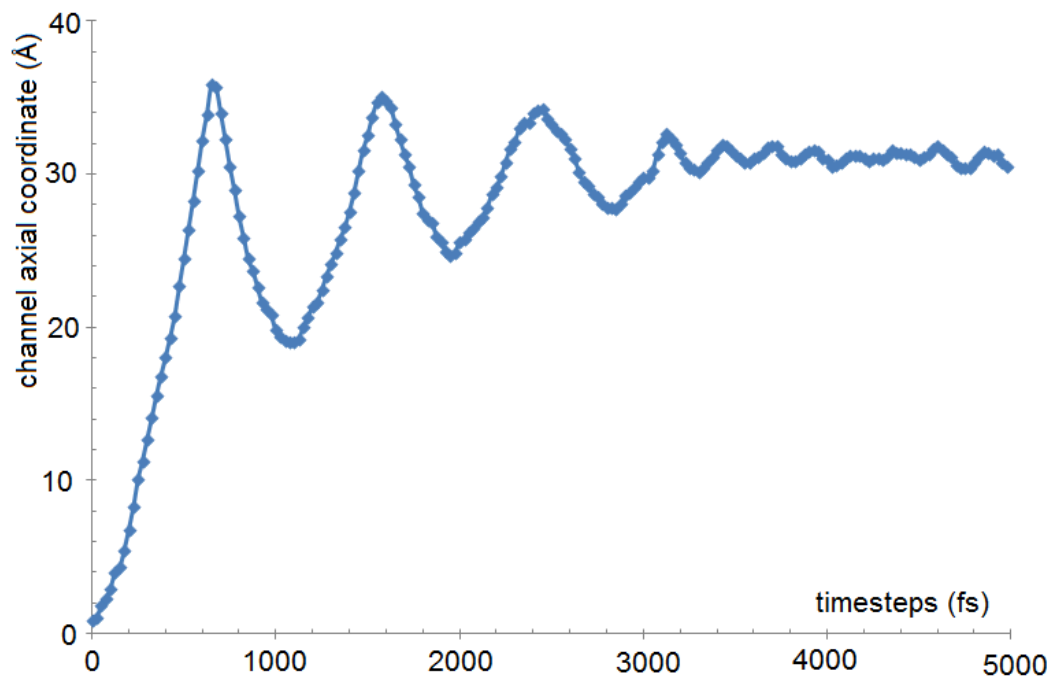

Fig. 7 The oscillating axial trajectory of a single $\mathrm{Na}^{+}$ion after entry to the channel in an electric field $(\varepsilon=0.3)$. The ion is simultaneously subject to this field and to the axial one generated by the atomic coulomb charges.

\section{Power}

The power $P$ emitted by an electric dipole $p$ oscillating with angular frequency $\omega$ radiates is given [17] by:

$$
P=\frac{p^{2}}{12 \pi \varepsilon_{0}} \frac{\omega^{4}}{c^{3}}
$$

In a quantum mechanical system, the power is expressed by an equation similar to (4) but in which the dipole $p$ is replaced by $p_{a b}^{z}=\iiint \psi_{a}^{*} z \psi_{b} d x d y d z$, which is the transition moment integral between states with wave functions $\psi_{a}$ and $\psi_{b}$ with polarization $z$. Since our model is a nonquantum one we use Eqn (4) in which the dipole $p$ results from the charge displacements of the $\mathrm{Na}^{+}$ ion as it oscillates along the $z$ axis of the channel. The $\varepsilon=0.5$ data that we considered in Fig. 5 
provides an amplitude of $30 \AA$ and $\omega=1.8 \times 10^{13} \mathrm{~Hz}$; these quantities lead to a power $P$ associated with the oscillations which is $2.7 \times 10^{-18} \mathrm{~J} \mathrm{~s}^{-1}$ from a single channel molecule.

A pulsed electric field?

A series of pulsed fields could presumably maintain the oscillations (damped by friction with the channel walls) indefinitely. Rather than deploy electric fields of the strengths described above the required field could be generated by irradiating the sample with pulsed radiation of the appropriate intensity and frequency.

The intensity of a beam is the time-averaged mean of the Poynting vector [17]:

$$
S=\varepsilon_{0} \varepsilon^{2} c
$$

where $\mathcal{E}$ and $\mathcal{Z}$ are the electric and magnetic field vectors associated with the beam, and are related by $\varepsilon=\boldsymbol{B}$ c. The magnitude of the Poynting vector is given by:

$$
S=\varepsilon_{0} \varepsilon^{2} c
$$

and the time-averaged intensity is:

$$
I=1 / 2 \varepsilon_{0} c\left(\boldsymbol{\varepsilon}_{\max }\right)^{2}
$$

which is the beam's power per unit area. A source of microwave radiation (provided by a highpower klystron) could deliver power up to $100 \mathrm{MW}$. Concentrating such a beam on to a $1 \mathrm{~cm}^{2}$ target would by Eqn (5) generate electric fields of $\sim 10^{8} \mathrm{v} \mathrm{m}^{-1}$ whose strengths are just an order of magnitude below those described here. The radiation emitted from the channel would contain frequencies similar, but not identical, to that of the exciting field and might be expected to contain information describing the ion and the channel.

\section{Exit from the channel?}

If the molecular channel is to mimic a membrane channel in a living cell, an ion must overcome the energy barrier at the end of the channel and exit into the extracellular fluid. In our ion channel system, raising the temperature allows the $\mathrm{Na}^{+}$migrant ion to reach a channel cavity further from the end at which it entered but (except at unrealistic temperatures!) does not facilitate its exit from the channel. The application of a moderate axial electric field brings the ion to a position in the channel from which the thermal excitation at temperatures above $360 \mathrm{~K}$ can effect its escape from into the surrounding solution (Fig. 8). At this point, it becomes strongly solvated and cannot re-enter the channel unless the direction of the electric field is reversed.

Owing to the degree of dynamic randomness in the mechanism of the $\mathrm{Na}^{+}$ion's desolvation before it enters the channel, the effect of temperature increase is not completely regular, but the overall trend is sensible. Fig. 8 shows the coincidence of the initial portions of the trajectory curves ( $7<z<37 \AA$ ) for the different temperatures; the ion channel migration is thus virtually nonactivated. Attempts to monitor velocities on an Arrhenius plot led to an activation energy of less than $1 \mathrm{~J} \mathrm{~mol}^{-1}$. 


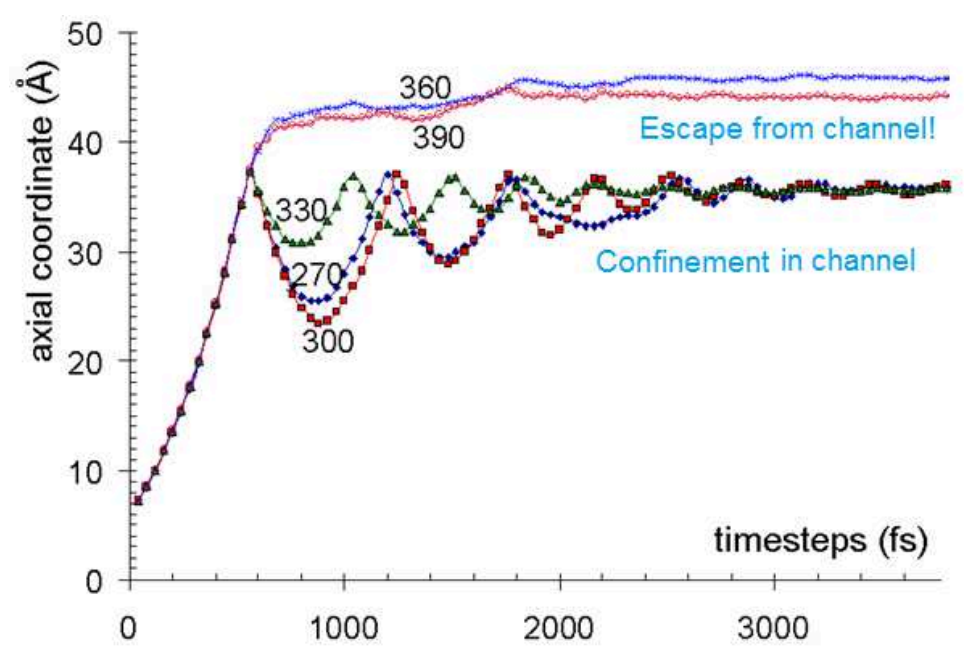

Fig. 8 Channel trajectory of a $\mathrm{Na}^{+}$ion in an electric field $\varepsilon=0.3 \mathrm{v} \AA^{-1}$ at the temperatures labelled. At the two highest temperatures the ion exits the channel.

\section{Damped oscillations}

The inelastic encounters between the ion migrant and the atoms constituting the inner surface of the channel result in a loss of energy by the ion as revealed by the rapid damping of the oscillations in Figs. 7 and 8. While it is not strictly correct to describe the resulting motion as a damped harmonic one, such a treatment has the advantage of simplicity and we shall adopt the harmonic model with the proviso that it is an approximation.

The damping is incorporated into the equation of motion by the addition of the term $k \dot{\zeta}$ proportional to the instantaneous velocity $\dot{\zeta}$ to describe the frictional deceleration of the oscillating particle. This term is commonly adopted to account for the friction of a macroscopic body in a fluid medium. For an ion of mass $m$ with angular frequency $\omega$ the equation then becomes:

$$
m \ddot{\zeta}+m k \dot{\zeta}+m \omega^{2}=0
$$

where $\zeta \equiv \zeta(t)$ is the ion's axial displacement at time $t$. Only one of the three limiting solutions [18] to Eqn. (6) describes the required damped oscillations:

$$
\zeta(t)=C^{e^{-3 / 2 k t} \cos \omega t}
$$

where $\tau=2 k^{-1}$ is the decay time constant. The oscillation and amplitude decays in Fig. 7 imply the parameters $\omega_{0}=0.018 \mathrm{fs}^{-1}$ and $k=10^{-3} \mathrm{fs}^{-1}$. The relaxation time $\tau=2 \times 10^{-12} \mathrm{~s}$ is consistent with the picosecond time scale derived from nanotribological measurements on sliding surfaces [19], and will be used in the next section to infer the frictional force in the inelastic interactions between the ions and the channel.

\section{Sliding friction of the $\mathrm{Na}^{+}$channel migration}

We investigate the sliding friction of an ion by examining the frictional force on the trajectory of an ion when it migrates along the channel with a range of velocities which are imposed by the application of electric fields of different strengths and directions.

The $\varepsilon=0.0$ trace in Fig. 9 shows that if there is no field then the ion's momentum carries it past the centre of the well before it occupies a halting site in the flat portion of the 


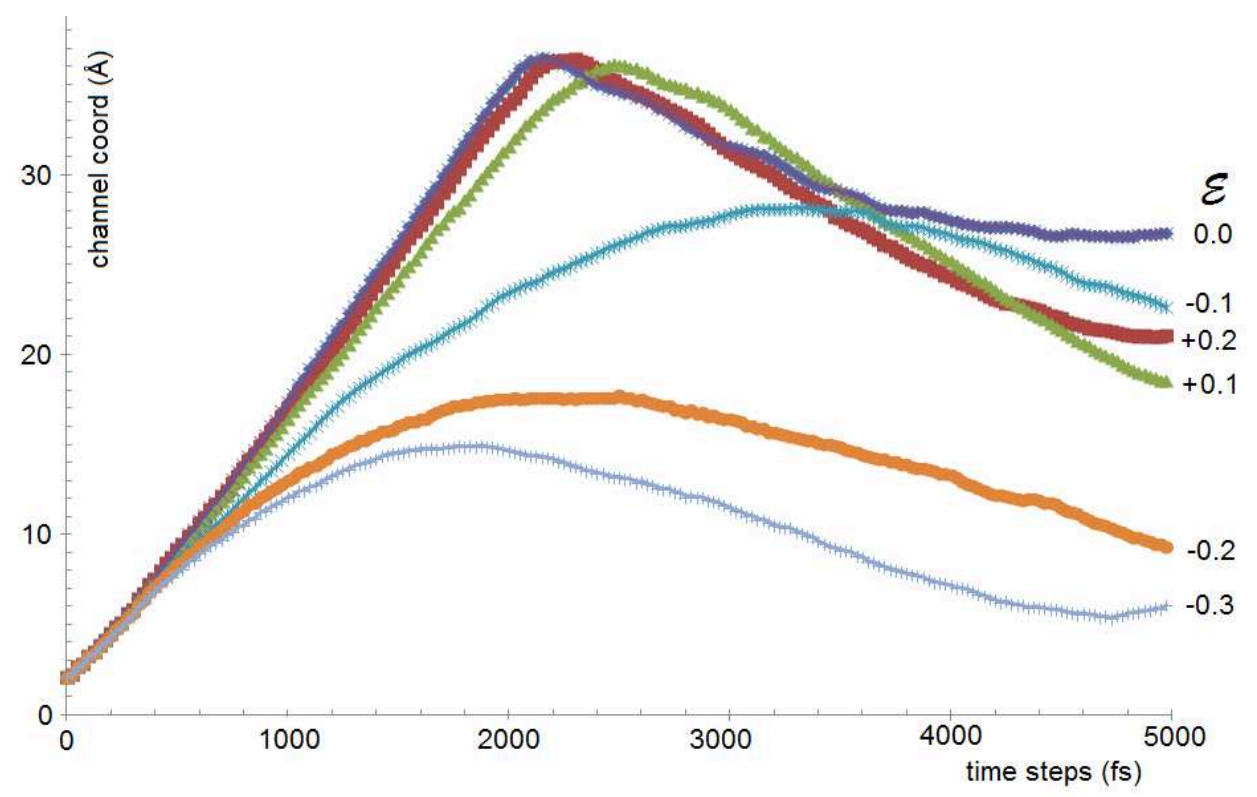

Fig. 9 Different values (positive and negative) of electric field $\varepsilon$ impart different trajectories and velocities to the migrating $\mathrm{Na}^{+}$.

potential. If the direction of the field opposes the effect of the Coulomb well $(\varepsilon<0)$ on entry, then the ion traverses the channel before returning from the constant-potential region to occupy a site on that side of the channel where it began its trajectory. The third case $(\varepsilon>0)$ is when the direction of the electric field is in the same sense as that of the coulombic potential after the ion has entered the channel. The mutual enhancement of the two driving forces makes the cation travel most of the length of the channel and to undergo trajectory oscillations, only the beginning of which are visible in Fig. 9.

Upon embarking on its trajectory a Na ${ }^{+}$ion rapidly accelerates to a velocity $1.26 \times 10^{3} \mathrm{~ms}^{-1}$ which is initially independent of the ion's position and of the strength and direction of the applied field. The existence of a constant-velocity region of the trajectories is consistent with the inclusion of the term $m k \dot{\zeta}$ to describe the frictional force that dampens the oscillations. This is the term that leads to a constant 'terminal velocity' imposed on a particle by the surrounding medium for example by the air or liquid on a falling body. For our $\mathrm{Na}^{+}$ion moving through the channel, the differing contributions by the coulomb well (at various migrant positions) and by the electric field (at the various imposed strengths and directions) lead to different ion velocities. The initial insensitivity of the ion's trajectory to the imposed field indicates that its dynamics are dominated by the electrostatic potential which are caused by the atoms within the channel i.e. the coulomb well. This is because the initial forces imposed by a $-12 \mathrm{v}$ potential greatly outweigh those from the imposed electric fields. As the ion falls into the coulombic well the presence of the $m k \dot{\zeta}$ frictional term ensures the maintenance of the $1.26 \times 10^{3} \mathrm{~ms}^{-1}$ terminal velocity until the force contributed by the level portion of the well is too small to provide the critical value and the motion is dominated by the friction. The stage at which this occurs depends on the 'positive or negative feedback' provided by the electric field that has been imposed as illustrated in Fig. 9.

On atomic scales the frictional force $f$ may not obey Amonton's Law which states that $f$ is independent of sliding velocity $v$ and may indeed depend on $v$ as we supposed in the previous section. Among molecular tribologists there is, however, little consensus on the details of this dependence. Although in Section 3.9 we explored the form $f \propto v$ used to describe the motion of macroscopic bodies, it must be admitted that friction force microscopy (where however the velocities are much greater than those here) finds a logarithmic dependence [20] while work on sliding polymer surfaces concludes that there is no dependence on $v$ [21]. We shall attempt to find a description of friction that is independent of the use of these forms. 


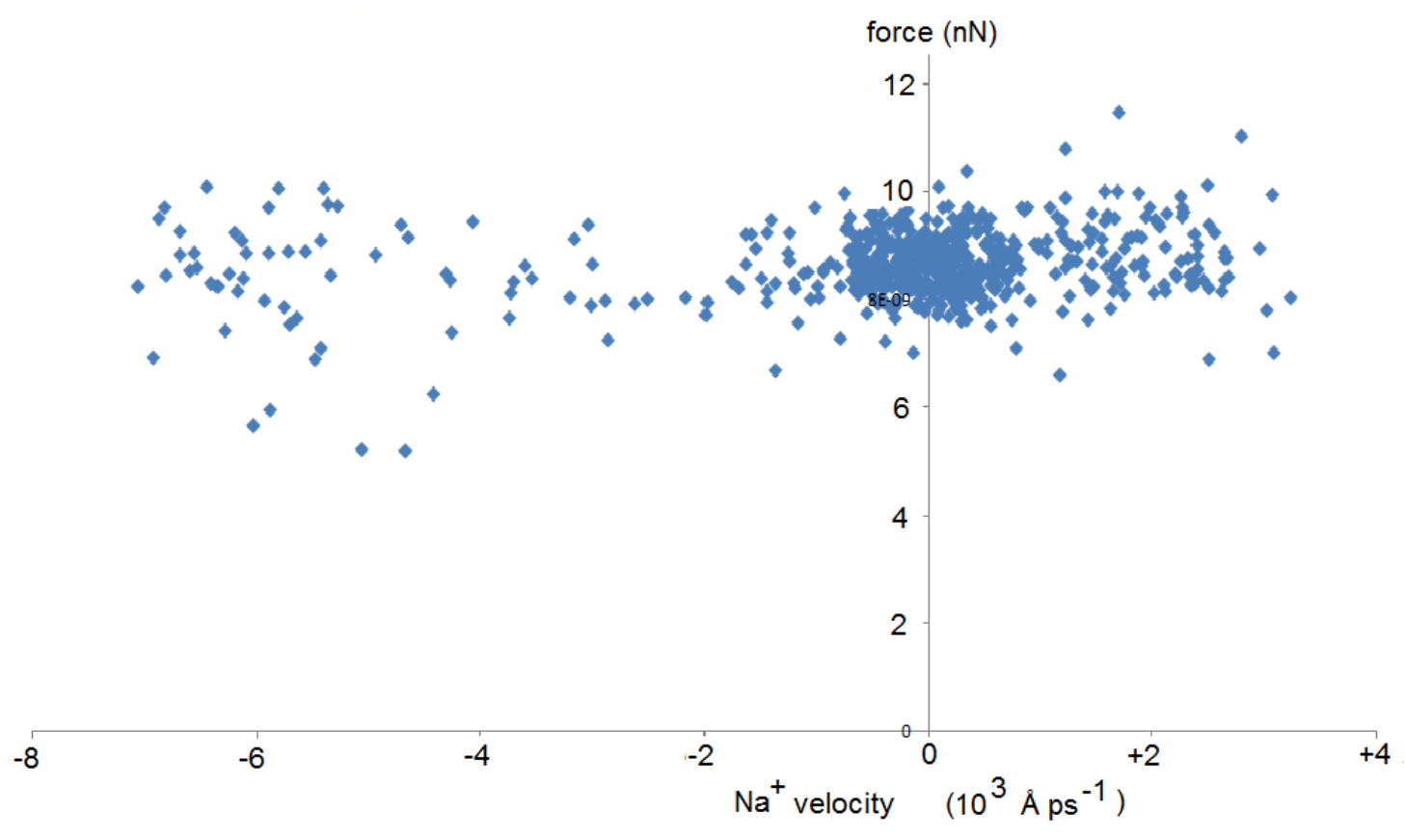

Fig. 10 The forces of sliding friction between the $\mathrm{Na}^{+}$ion and the channel. The velocities are taken from the data used to construct Fig. 9.

However the danger in oversimplification of the frictional theory is apparent when trying to make a direct calculation of the frictional force on a cation at a any point on the axial coordinate. This was done as follows. When $\mathrm{Na}^{+}$is at any position $z$ in the channel the total force $F_{\text {tot }}$ on the ion and its velocity are computed explicitly by the molecular dynamics calculation that are then used to determine the velocity and acceleration vectors for the subsequent time-step. The frictional force on the ion is obtained by subtracting from $F_{t o t}$ the coulombic force $F_{c}$ on the ion that is generated by the channel atoms:

$$
F_{c}(z)=\sum_{i=1}^{14} \sum_{j=1}^{n}\left[\frac{q_{j}}{r^{2}+\left(p_{i}+z\right)^{2}}\right]
$$

where, as in Eqn. (1), $n$ is the number of atoms in each of the 14 component CE rings, $r_{j}$ is the distance of charge $q_{j}$ from the axis in the ring and $p_{\mathrm{i}}$ is the axial position of the $i^{\text {th }}$ ring. The attainment of equilibrium for these short-time events was optimised by shortening the time-step unit from the usual $1 \mathrm{fs}$ to $0.1 \mathrm{fs}\left(10^{-16} \mathrm{~s}\right)$. The friction could thus be tested at each time-step for a wide range of velocities. These were generated by subjecting the $\mathrm{Na}^{+}$ion to various electric fields applied in both directions in turn along the channel $\left[-0.3<\varepsilon<+0.3 \mathrm{v} \AA^{-1}\right]$. This imposes a range of velocities on the ion that are plotted in Fig. 10. Despite the scattered nature of the plots (the result of velocity corrections from the coupled thermostat at high ion velocities) the Figure shows that the friction seems appreciably constant at $8-9 \mathrm{nN}$ over the velocity range of 0 to $1 \times 10^{3} \mathrm{~nm} \mathrm{~s}^{-1}$ which would seem to require that the $f \propto v^{n}$ relation is obeyed only for $n=0$. That ionic friction in the molecular system should be independent of sliding velocity is inconsistent with our assumption, in an earlier section, of the term $m k \dot{\zeta}$ to describe the frictional damping force of the oscillations. Although at that point attention was called to the approximation of the harmonic form for the ion oscillation and to the exercise of caution in the interpretation of the results, it is unlikely that the discrepancy follows simply from the non-harmonicity of the oscillations. It is possible that a more 
realistic force should be assigned a less simple form than $f \propto m k \dot{\zeta}$. Nevertheless from the derived value $k=10^{-3} \mathrm{fs}^{-1}$, the mass $3.84 \times 10^{-26} \mathrm{~kg}$ of $\mathrm{Na}^{+}$and its typical velocity $500 \mathrm{~ms}^{-1}$ molecular dynamics the force $m k \dot{\zeta}$ is of the order of $10 \mathrm{nN}$, which is practically the same as the value calculated from the sliding friction.

\section{The effect of channel rigidity}

Natural ion channels in cell membranes and most of the synthetic ones that were cited in Sections 1 and 2.1 possess conformational freedom. Thus we should like to see how the ion transport character is changed when the rigidity of the channel is modified. Accordingly we consider 14-ring channels similar to the one defined in Fig. 1 for the investigations reported above, but where elements are introduced to increase the flexibility of the channel. The rigidity of the former channel was due to the presence of the ten $>\mathrm{CH}_{2}-\mathrm{NH}-\mathrm{CH}_{2}<$ molecular 'brackets' connecting each $\mathrm{CE}$ ring to the adjacent one (A in Fig. 11) compares this structure to $\mathbf{B}$ in which the bracket has been removed and an aza group $>\mathrm{C}-\mathrm{O}-\mathrm{NH}-\mathrm{C}<$ included directly as part of the crown ether ring. As the number of inter-ring linking groups is thereby reduced from 10 to 5 a more flexible molecule is created.

\section{Ring dynamics}

In this part of the investigation again no membrane was present and rigid channels $\mathbf{A}$ and flexible channel $\mathbf{B}$ floated freely in aqueous solutions of $\mathrm{LiF}, \mathrm{NaCl}$ or $\mathrm{KCl}$. The incursion of molecular species into $\mathbf{B}$ from outside the channel was examined by monitoring their positions and rdf traces as described for $\mathbf{A}$ in Sections 3.2 and 3.3. As we saw to be the case also for the rigid channel $\mathbf{A}$, cations $\mathrm{Li}^{+}, \mathrm{Na}^{+}$and $\mathrm{K}^{+}$were admitted to $\mathbf{B}$ but anions or solvent water molecules could not be. The molecular geometry of $\mathbf{B}$ 's inner ring is similar to that of $\mathbf{A}$ as also is the axial coulomb potential of $\mathbf{B}$ to that of $\mathbf{A}$ (Fig. 2). We may therefore use the same arguments to conclude that entry of ions into either of them is governed principally by the electrical charges of the atoms in the channel's interior.

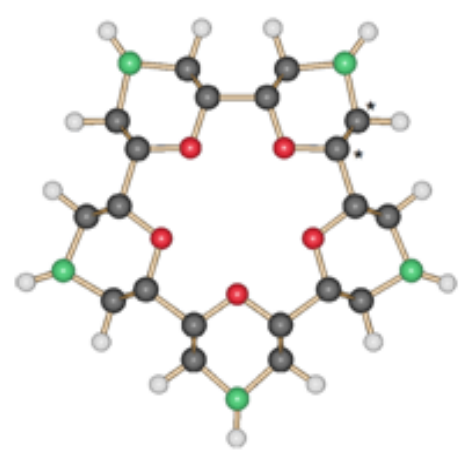

A

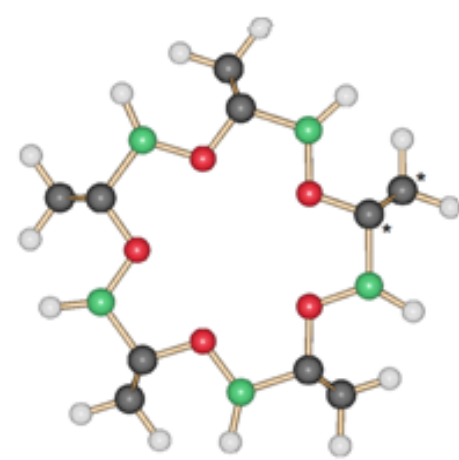

B

Fig. 11 Modifications of the CE rings in the channel. The two structures define the constituent rings of channels of differing rigidities. A is the rigid channel of Fig. 1, while in $\mathbf{B}$ there are fewer interring links and the conformational structure is looser. The atom colour coding and the marked link atoms are as explained in Fig. 1.

In a marked contrast to the behaviour of the ions in channel $\mathbf{A}$, however, molecular dynamics showed that the cations were admitted much more readily into the flexible channel $\mathbf{B}$ than that to A. For example whereas electric fields of $0.1-0.4 \mathrm{v} \AA^{-1}$ were required to initiate cation entry 
into $\mathbf{A}$ an $\mathrm{Na}^{+}$ion placed within $\sim 1.0 \AA$ would usually enter the $\mathbf{B}$ channel unaided by a field. This implies that the greater flexibility of the first CE ring of $\mathbf{B}$ compared with that of the first ring in $\mathbf{A}$ alleviates the restrictive impediment imposed by its $4.0 \AA$ diameter. Of course since the cation does not bring its solvated water molecules into the channel the contribution to the energy barrier in this region that results from the necessary desolvation of the cation is still present.

Let us try to quantify the different behaviour of the $\mathrm{Na}^{+}$ion as it migrates in the two species of molecular channel by considering the motions associated with the CE rings in each of them. The dynamics will first be monitored by following the average time-dependence of the torsional angles involving the ether $\mathrm{O}$ atoms in each of the $14 \mathrm{CE}$ rings in $\mathbf{A}$ and $\mathbf{B}$ in Fig. 11; these are the atoms whose charges are largely responsible for the axial $-12 \mathrm{v}$ electrostatic potential. In order to monitor the bond torsions we select two sequential atom quartets and calculate the dihedral angles $\varphi$ defined by them. In the $\mathrm{CE}$ ring of rigid channel $\mathbf{A}$ they are $\mathrm{C}-\mathrm{C}-\mathrm{O}-\mathrm{C}$ and in the $\mathbf{B}$ channel C-N-O-C. Torsions derived from these would then reflect the motions of the ether oxygen atoms relative to the ring carbons. (The dynamics of other atom quartets were also investigated, but they are not reported as they led to similar conclusions.) Electric fields were not applied for this study but since a spontaneous entry of $\mathrm{Na}^{+}$from the bulk solution would be effected to $\mathbf{B}$ but not to $\mathbf{A}$, for a fair comparison of the two the cations were placed just inside the terminal cavity that is bounded by the five $\mathrm{OH}$ groups of the first rings referred to in Section 2.1 and shown in Fig. 1(c). This obviates a necessity to overcome the desolvation energy barrier.

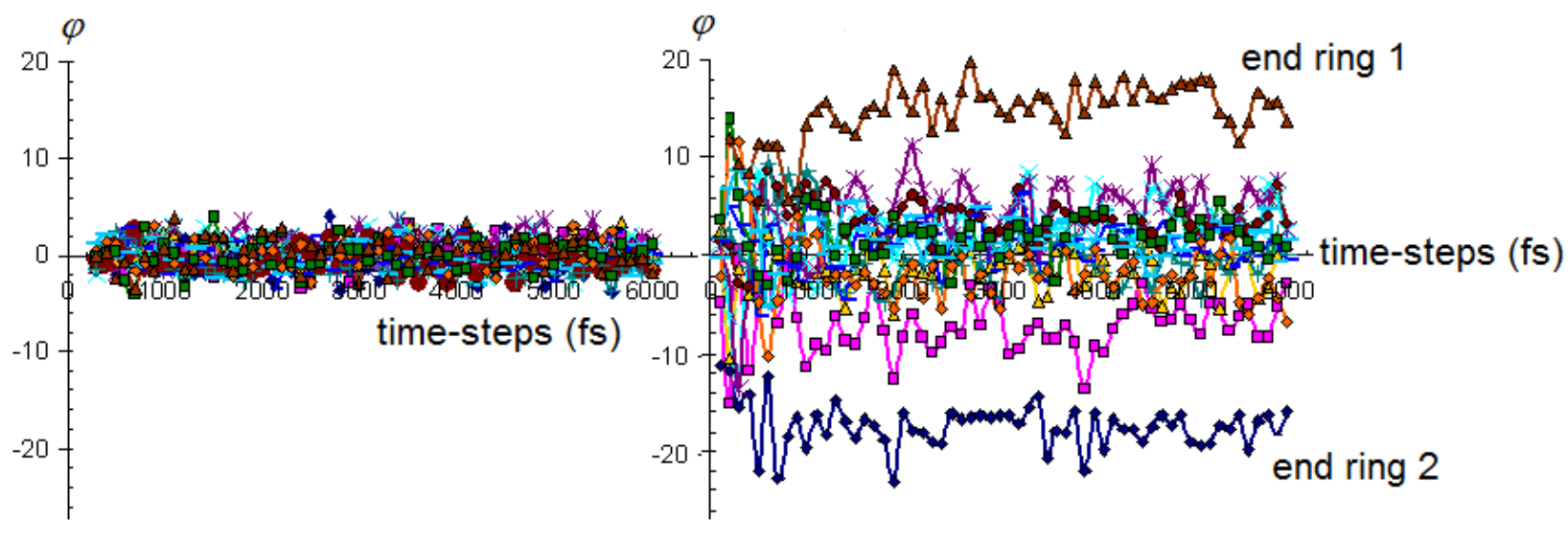

rigid $(A)$

flexible (B)

Fig. 12 Torsional angles to illustrate the natural out-of plane distortions in the crown ether rings in channels $\mathbf{A}$ and $\mathbf{B}$ defined in Fig. 11. The dihedral angles of the $\mathrm{C}-\mathrm{C}-\mathrm{O}-\mathrm{C}$ quartet of atoms in $\mathbf{A}$ and the C-N-O-C in $\mathbf{B}$ emphasise the flexibility of the $\mathbf{B}$ channel.

The average torsional changes in the rings as measured by the dihedral angle $\varphi$ were monitored to see if they responded to the motion of a $\mathrm{Na}^{+}$ion migrating along the axis as described in the previous paragraph. A comparison of the $\varphi$ spread for the two species of channel in $\mathbf{A}$ and $\mathbf{B}$ (in the absence of a Na${ }^{+}$migrant) in Fig. 12 demonstrates two important effects of the $\mathrm{CH}-\mathrm{NH}-\mathrm{CH}$ bracket on the ring torsions - the ranges of the $\varphi$ angles and the amplitudes of their timefluctuations. The contrast is made clear by plotting the very different $\varphi$ ranges in $\mathbf{A}$ and $\mathbf{B}$ with the same scale. In the rigid channel $\mathbf{A}$ the $\varphi(t)$ values demonstrate the securing effect of the $\mathrm{CH}-\mathrm{NH}-\mathrm{CH}$ brackets between the rings; the figure shows that the distortions from planarity in the CE rings of the rigid channel is very slight (only a few degrees). The greater spread of $\varphi(t)$ values in the flexible channel $\mathbf{B}$ shows that its rings distort more readily; significantly the end rings (1 and 14) show torsions of up to about $20^{\circ}$ and the inner rings of up to $10^{\circ}$, suggesting that the out-of-plane distortions of the end rings are an important factor to facilitate the admission of $\mathrm{Na}^{+}$ions. 
Fig. 13 monitors the five torsional angles $\varphi_{1}, \varphi_{2}, \ldots, \varphi_{5}$ of a single CE ring in the centre of the channel during the migration of a $\mathrm{Na}^{+}$. It shows how ring $\mathrm{N}^{\circ} .6$ responds to the ion's passage during the $0<t<1000$ ts time interval; upon the arrival of the ion, the ring adjusts its $-\mathrm{C}-\mathrm{N}-\mathrm{O}-\mathrm{C}$ dihedral angles. Although $\varphi(t)$ values for only the $6^{\text {th }}$ ring are displayed in the Figure they were essentially similar for all the 12 inner rings, demonstrating the rings' out-of plane buckling responses to the passage of the $\mathrm{Na}^{+}$in the 5 units in the defined time interval. Inspection of the atom coordinates shows that the direction of the change in $\varphi$ is that in which the ether oxygen atoms move towards the approaching $\mathrm{Na}^{+}$ion, suggesting that these changes are due to the coulombic attraction between the cation charge and the partial charges on the neighbouring ring ether oxygen atoms.
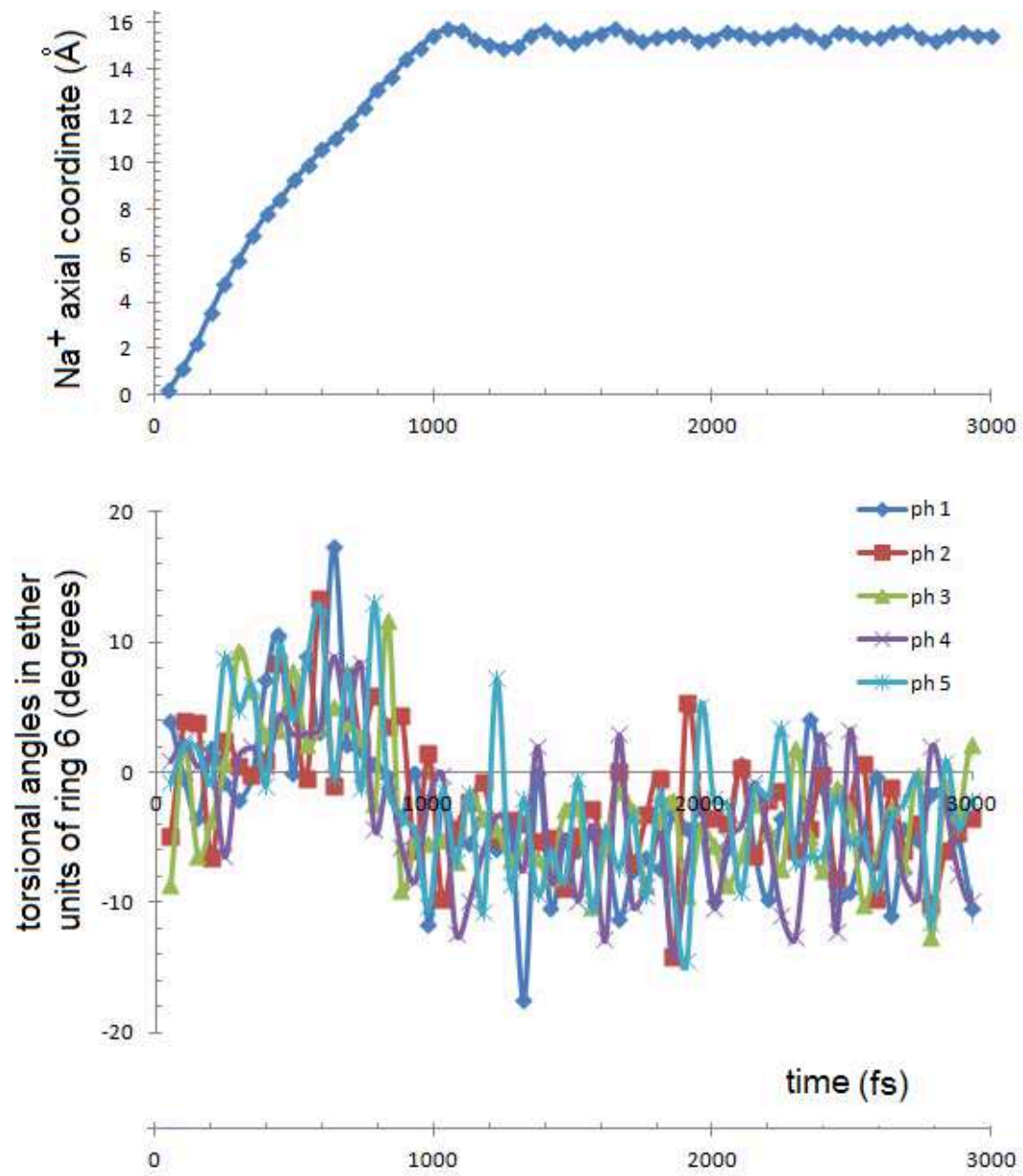

Fig. 13 Changes in the dihedral angles (lower) of the 5 C-N-O-C groups in the $6^{\text {th }} \mathrm{CE}$ ring in the $\mathbf{B}$ channel as $\mathrm{Na}^{+}$ion migrates along its axis (upper).

\section{Conclusions and discussion}

If mechanical devices are to be designed to operate on a nano scale such as those described in ref. [2] we shall need to understand in detail the mechanisms describing how the molecular components respond to user-imposed conditions, and the degree to which the response approaches that of a macroscopic machine. The component systems in this work are sufficiently simple to control their simulation conditions and to interpret the results. 
We have shown that the conductivity of ions in a channel composed of stacked 15-crownether-5 rings that is immersed in an aqueous electrolyte solution depends on (i) the charges on the atoms in the interior of the channel and (ii) the manner in which the rings are mutually linked. In a previous study of migration through a channel connecting two aqueous media across a bilayer membrane [16] each pair of adjacent $\mathrm{CE}$ rings was connected by five methylene $\left(\mathrm{CH}_{2}\right)$ groups. In such a system molecular dynamics predicted that a consequence of the ease of the deformation of the rings was that not only cations $\mathrm{Li}^{+}, \mathrm{Na}^{+}$, and $\mathrm{K}^{+}$could migrate in the channel, but the application of axial electric fields enabled also some solvent molecules (water) to enter the channel by an electrophoresis-like mechanism in which they were 'dragged in' by the cations.

We investigated the effect of condition (ii) in the previous paragraph when the five 'molecular brackets $>\mathrm{CH}-\mathrm{NH}-\mathrm{CH}<$ ' connected each $\mathrm{CE}$ ring to its neighbour. The enhanced rigidity of the resulting channel in comparison with one without such clamping brackets showed that its ability for the spontaneous admission of cations was lost. The ions could now only enter the channel with the aid of an imposed axial electric field. The transport of the ions described in this work is passive in the sense that its efficacy does not require significant configurational or conformational changes in the carrier species as occur in the active biological trans-membrane processes in living cells. Nevertheless the requirement of the carrier to respond to the presence of a migrant has been shown in a series of channels: from the highly flexible channel described in ref. [16] which admitted both cations and solvent molecules, to the unbracketed channel $\mathbf{B}$ in Section 3.12 of this work which permitted the entrance of only cations, to the rigid channel $\mathbf{A}$ which admitted cations only on the application of a suitable electric field.

A pulsed terahertz field would regenerate the oscillations which, for the $\varepsilon=0.3$ case referred to in this work, has a power output of the order of $10^{-18} \mathrm{~W}$ for each resonating channel molecule in the system. If the molar concentration of these channels were $0.001 \mathrm{M}$, the generated radiation from a $10 \mathrm{~cm}^{3}$ sample in which all the channels were aligned in the direction of the applied pulsed electric field, the sample would have a maximum power of $16 \mathrm{w}$. Now if the molecular solute in a low viscosity solution has a tumbling relaxation time of the order of $10^{-10} \mathrm{~s}$ a given channel should present any direction to an externally applied one on the same $0.1 \mathrm{~ns}$ timescale that has been described for the system, when there would be a sufficient number of solute molecules at optimally oriented at any instant for the ionic oscillation to occur. The behaviour of the cations after their (field-assisted) admission to the rigid channel would exhibit a spectrum at microwave to infra red frequencies containing details of the environment of the oscillating ions.

The design of nano-mechanical devices would require optimisations of a large number of components and conditions. As well as the operating temperature and pressure, factors to be controlled would be the sizes and charges of the migrant species and their velocities and precise direction in which the ions enter the channel. The effects of the shape and rigidity of the channel can be investigated through the atomic species constituting it, which in turn define the charges on the atoms of the interior and the interactions of the latter with the migrant species. Some of the importance that was attached in this work to the issue of channel rigidity arose from the established role played by phonons in charge transport, e.g. in DNA chains [22], a feature to consider will be a non-rigid channel whose conformational motions and phonon modes may play an active part in the transport of migrants.

Attention was drawn to the problem of assigning a velocity exponent to the frictional force relation $f \propto v^{n}$ that can describe sliding frictional forces on a molecular scale. However both approaches described in this work led to forces of order $\sim 1 \mathrm{nN}$. It is clear that rather than attempting to fit theories developed for the dynamics of macroscopic bodies a more precise description of the tribological interactions of molecular particles on the nanoscale is required.

\section{Computational facilities}

An Institúd um Theicneolaíocht Eolais agus Riomhfhorbairt na hÉireann (IITAC). 


\section{References}

[1] D.J. Aidley and P.R. Stanfield, Ion Channels, Cambridge University Press, Cambridge 1996.

[2] J.-C. Olsen, K. E. Griffiths and J. F. Stoddart , 'A Short History of the Mechanical Bond', in From Non-Covalent Assemblies to Molecular Machines, J.-P. Sauvage, P. Gaspard (Eds.), WileyVCH: Weinheim, Germany (2011), pp 67-139.

[3](see for example) B. Roux, B Prod'hom, M. Karplus, Biophys J. 68 pp. 876-892 (1995).

[4] S. Matile, A. Som and N. Sorde, Tetrahedron 60 6405-35 (2004); N. Sakai and S. Matile, Langmuir 29 9031-40 (2013); T.M. Fyles, Chem. Soc. Rev. 36 335-47 (2007); L.Echegoyen, Nature 369 276-7 (1994).

[5] I.Tabushi, Y.Kuroda and K.Yokota, Tetrahedron Lett. 23 4601-4 (1982)].

[6] M.R. Ghadiri, J.R. Granja and Lukas K. Buehler, Nature 369301 (1994).

[7] G.A. Woolley and B.A Wallace, J. Membr. Biol. 129 109-136 (1992)].

[8] G.W. Gokel, R.Ferdani, J.Liu, R.Pajewski, H. Shabany and P. Uetrecht, Chem.-Eur. J. 7 33-39 (2001)].

[9] D.A. Morton-Blake, Journal of Molecular Liquids 167, 57 (2012).

[10] W. Smith and T.R. Forester, J. Molec. Graphics 14 (1996) 136.

[11] Gaussian 03, Revision E.01, M. J. Frisch, G. W. Trucks, H. B. Schlegel, G. E. Scuseria, M. A. Robb, J. R. Cheeseman, J. A. Montgomery, Jr., T. Vreven, K. N. Kudin, J. C. Burant, J. M. Millam, S. S. Iyengar, J. Tomasi, V. Barone, B. Mennucci, M. Cossi, G. Scalmani, N. Rega, G. A. Petersson, H. Nakatsuji, M. Hada, M. Ehara, K. Toyota, R. Fukuda, J. Hasegawa, M. Ishida, T. Nakajima, Y. Honda, O. Kitao, H. Nakai, M. Klene, X. Li, J. E. Knox, H. P. Hratchian, J. B. Cross, V. Bakken, C. Adamo, J. Jaramillo, R. Gomperts, R. E. Stratmann, O. Yazyev, A. J. Austin, R. Cammi, C. Pomelli, J. W. Ochterski, P. Y. Ayala, K. Morokuma, G. A. Voth, P. Salvador, J. J. Dannenberg, V. G. Zakrzewski, S. Dapprich, A. D. Daniels, M. C. Strain, O. Farkas, D. K. Malick, A. D. Rabuck, K. Raghavachari, J. B. Foresman, J. V. Ortiz, Q. Cui, A. G. Baboul, S. Clifford, J. Cioslowski, B. B. Stefanov, G. Liu, A. Liashenko, P. Piskorz, I. Komaromi, R. L. Martin, D. J. Fox, T. Keith, M. A. Al-Laham, C. Y. Peng, A. Nanayakkara, M. Challacombe, P. M. W. Gill, B. Johnson, W. Chen, M. W. Wong, C. Gonzalez, and J. A. Pople, Gaussian, Inc., Wallingford CT, 2004.

[12] J. Åqvist, J. Phys. Chem. 94 8021-8024 (1990).

[13] S.H. Lee and J.C. Rasaiah, J. Chem. Phys. 1001420 (1996).

[14] S. L. Mayo, B. D. Olafson and W.A. Goddard III, J. Phys. Chem. 94 (1990) 8897-8909.

[15] H. J. C. Berendsen, J. R. Grigera, and T. P. Straatsma, J. Phys. Chem. 91 (1987), pp. 62696271.

[16] D.A. Morton-Blake, Soft Matter 6558 (2010).

[17] H.D. Young, R.A. Freedman, University Physics $11^{\text {th }}$ ed., Pearson Addison Wesley, San Francisco 2004. 
[18] D.A. Morton-Blake and Conan Kumari-Doyle, Computational and Theoretical Chemistry 1008 74 (2013).

[19] L. Singer, Friction and energy dissipation at the atomic scale: A review. J. Vac. Sci. Technol. A (1994) 12(5), Sep/Oct 2605-216; Fundamentals of Friction, edited by I. L. Singer and H. M. Pollock $\sim$ Kluwer, Dordrecht, 1992.

[20] E. Gnecco, R. Bennewitz, T. Gaylog, Ch. Loppacher, M. Bammerlin, E. Meyer, H.-J. Güntherodt, Physical Review Letters 841172 (2000).

[21] I.M. Sivebaek, V.N. Samoilov, Bo.N. J. Persson, Langmuir 268721 (2010).

[22] P.T. Henderson, D. Jones, G. Hampikian, Y. Kan, G.B. Schuster,Proc. Natl. Academy of Sciences of the United States of America 96, 8353-8358 (1999). 